TRANSACTIONS OF THE

AMERICAN MATHEMATICAL SOCIETY

Volume 348, Number 11, November 1996

\title{
KRULL-SCHMIDT FAILS FOR SERIAL MODULES
}

\author{
ALBERTO FACCHINI
}

\begin{abstract}
We answer a question posed by Warfield in 1975: the KrullSchmidt Theorem does not hold for serial modules, as we show via an example. Nevertheless we prove a weak form of the Krull-Schmidt Theorem for serial modules (Theorem 1.9). And we show that the Grothendieck group of the class of serial modules of finite Goldie dimension over a fixed ring $R$ is a free abelian group.
\end{abstract}

In 1975 R. B. Warfield published a very interesting paper [8], in which he described the structure of serial rings and proved that every finitely presented module over a serial ring is a direct sum of uniserial modules. On page 189 of that paper, talking of the problems that remained open, he said that "... perhaps the outstanding open problem is the uniqueness question for decompositions of a finitely presented module into uniserial summands (proved in the commutative case and in one noncommutative case by Kaplansky [5])." We solve Warfield's problem completely: Krull-Schmidt fails for serial modules.

The two main ideas in this paper are the epigeny class and monogeny class of a module. We say that modules $U$ and $V$ are in the same monogeny class, and we write $[U]_{m}=[V]_{m}$, if there exist a module monomorphism $U \rightarrow V$ and a module monomorphism $V \rightarrow U$. In the same spirit, we say that $U$ and $V$ are in the same epigeny class, and write $[U]_{e}=[V]_{e}$, if there exist a module epimorphism $U \rightarrow V$ and a module epimorphism $V \rightarrow U$. The significance of these definitions is that uniserial modules $U, V$ are isomorphic if and only if $[U]_{m}=[V]_{m}$ and $[U]_{e}=[V]_{e}$ (Proposition 1.6). Our technical starting point is that the endomorphism ring of a uniserial module has at most two maximal ideals, and modulo those ideals it becomes a division ring (Theorem 1.2).

We show (Theorem 1.9) that if $U_{1}, \ldots, U_{n}, V_{1}, \ldots, V_{t}$ are non-zero uniserial modules, then $U_{1} \oplus \cdots \oplus U_{n} \cong V_{1} \oplus \cdots \oplus V_{t}$ if and only if $n=t$ and there are two permutations $\sigma, \tau$ of $\{1,2, \ldots, n\}$ such that $\left[U_{\sigma(i)}\right]_{m}=\left[V_{i}\right]_{m}$ and $\left[U_{\tau(i)}\right]_{e}=\left[V_{i}\right]_{e}$ for every $i=1,2, \ldots, n$. And we show that for every $n \geq 2$ there exist $2 n$ pairwise non-isomorphic finitely presented uniserial modules $U_{1}, U_{2}, \ldots, U_{n}, V_{1}, V_{2}, \ldots$, $V_{n}$ over a suitable serial ring such that $U_{1} \oplus U_{2} \oplus \cdots \oplus U_{n} \cong V_{1} \oplus V_{2} \oplus \cdots \oplus V_{n}$ (Example 2.2).

The weakened form of the Krull-Schmidt Theorem that serial modules satisfy (Theorem 1.9) is sufficient to allow us to compute the Grothendieck group of the class of serial modules of finite Goldie dimension over a fixed ring $R$. As is well

Received by the editors August 4, 1995.

1991 Mathematics Subject Classification. Primary 16D70, 16S50, 16P60.

Partially supported by Ministero dell'Università e della Ricerca Scientifica e Tecnologica (Fondi $40 \%$ e $60 \%$ ), Italy. This author is a member of GNSAGA of CNR.

(C)1996 American Mathematical Society 
known, if the Krull-Schmidt Theorem holds for a certain class of modules, its Grothendieck group is a free abelian group. Though the Krull-Schmidt Theorem does not hold for the class of serial modules of finite Goldie dimension, its Grothendieck group is a free abelian group. The Krull-Schmidt Theorem fails because the Grothendieck group is free as an abelian group, but it is not order isomorphic to a free abelian group with the pointwise order (Section 3.2).

There is a vague resemblance between the behavior of serial modules and that of artinian modules. For instance, in Section 3 we show that endomorphism rings of serial modules of finite Goldie dimension are semilocal rings, that is, they are semisimple artinian modulo their Jacobson radical. Camps and Dicks proved that endomorphism rings of artinian modules also are semilocal [1]. Here we prove that Krull-Schmidt fails for serial modules. In [2] Herbera, Levy, Vámos and the author proved that Krull-Schmidt fails for artinian modules, thus answering a question posed by Krull in 1932.

The author thanks Larry Levy and the referee for some most useful suggestions on previous versions of this paper.

We shall consider right unital modules over an associative ring $R$ with $1 \neq 0$. A module is uniserial if its lattice of submodules is linearly ordered under inclusion, and is a serial module if it is a direct sum of uniserial modules. A ring is serial if it is a serial module both as a right module and as a left module over itself. The symbol $\subset$ will denote proper inclusion, and, if $S$ is a ring, $J(S)$ will denote the Jacobson radical of $S$.

A serial module is of finite Goldie dimension if and only if it is the direct sum of a finite number of uniserial modules. More precisely, a serial module $M$ has finite Goldie dimension $n$ if and only if it is the direct sum of $n$ non-zero uniserial modules, so that the number $n$ of direct summands of $M$ that appear in any decomposition of $M$ as a direct sum of non-zero uniserial modules does not depend on the decomposition.

\section{Monogeny AND EPIGENy}

The following elementary lemma will often be useful in the sequel.

Lemma 1.1. Let $A, C$ be non-zero right modules over an arbitrary ring $R, B$ a uniserial right $R$-module and $\alpha: A \rightarrow B, \beta: B \rightarrow C$ homomorphisms. Then

(a) $\beta \alpha$ is a monomorphism if and only if $\beta$ and $\alpha$ are both monomorphisms;

(b) $\beta \alpha$ is an epimorphism if and only if $\beta$ and $\alpha$ are both epimorphisms.

Proof. (a) We must prove that if $\beta \alpha$ is a monomorphism, $\beta$ also is a monomorphism. Now if $\beta \alpha$ is a monomorphism, then $\alpha(A) \cap \operatorname{ker}(\beta)=0$. Since $B$ is uniserial, either $\alpha(A)=0$ or $\operatorname{ker}(\beta)=0$. Now $\alpha(A)=0$ implies $\beta \alpha=0$, and this is not a monomorphism because $A \neq 0$. Hence $\operatorname{ker}(\beta)=0$.

(b) We must prove that if $\beta \alpha$ is an epimorphism, $\alpha$ also is an epimorphism. Now if $\beta \alpha$ is an epimorphism and $C \neq 0$, then $\beta \alpha \neq 0$, so that $\beta \neq 0$. Hence $\operatorname{ker}(\beta) \subset B$. If $\alpha(A) \subset B$, then $\operatorname{ker}(\beta)+\alpha(A) \subset B$. Now $\beta$ induces a one-to-one order preserving mapping between the set of all submodules of $B$ containing $\operatorname{ker}(\beta)$ and the set of all submodules of $\beta(B)$. Hence $\operatorname{ker}(\beta)+\alpha(A) \subset B$ implies $\beta(\operatorname{ker}(\beta)+\alpha(A)) \subset \beta(B)$, that is, $\beta \alpha(A) \subset \beta(B) \subseteq C$. Hence $\beta \alpha$ is not an epimorphism, a contradiction. This proves that $\alpha(A)=B$ and $\alpha$ is an epimorphism. 
Theorem 1.2. Let $A_{R}$ be a non-zero uniserial module and $E=\operatorname{End}\left(A_{R}\right)$ its endomorphism ring. Let $I$ be the subset of $E$ consisting of all the endomorphisms of $A_{R}$ that are not monomorphisms, and $J$ be the subset of $E$ consisting of all the endomorphisms of $A_{R}$ that are not epimorphisms. Then $I$ and $J$ are completely prime two-sided ideals of $E$, every right (or left) proper ideal of $E$ is contained either in $I$ or in $J$, and either

(a) the ideals $I$ and $J$ are comparable, so that $E$ is a local ring with maximal ideal $I \cup J$, or

(b) the ideals $I$ and $J$ are not comparable, $I \cap J$ is the Jacobson radical $J(E)$ of $E$, and $E / J(E)$ is canonically isomorphic to the direct product $E / I \times E / J$ of the two division rings $E / I$ and $E / J$.

Proof. Obviously $I$ and $J$ are additively closed. They are two-sided completely prime ideals of $E$ by Lemma 1.1.

Let $K$ be an arbitrary proper right or left ideal of $E$. Since $I \cup J$ is exactly the set of non-invertible elements of $E$, it follows that $K \subseteq I \cup J$. But then either $K \subseteq I$ or $K \subseteq J$. (Otherwise there exist $x \in K \backslash I$ and $y \in K \backslash J$. Then $x+y \in K$, $x \in J$, and $y \in I$. Thus $x+y \notin I$ and $x+y \notin J$. Hence $x+y \notin I \cup J$. This is a contradiction because $K \subseteq I \cup J$.)

Thus every proper right or left ideal of $E$ is contained either in $I$ or in $J$. Therefore the unique maximal right ideals of $E$ are at most $I$ and $J$, and similarly for left ideals. If $I \subseteq J$ or $J \subseteq I$, then $E$ is local ring with maximal ideal $I \cup J$ and case (a) holds. Otherwise $I$ and $J$ are the two unique maximal right ideals of $E$. Therefore $I \cap J$ is the Jacobson radical of $E$ and hence there is a canonical injective ring morphism $E / J(E) \rightarrow E / I \times E / J$. Since $I+J=R$, this ring morphism is onto by the Chinese Remainder Theorem.

Corollary 1.3. Uniserial modules cancel from direct sums, that is, if $A$ is a serial module of finite Goldie dimension and $B, C$ are arbitrary modules, then $A \oplus B \cong$ $A \oplus C$ implies $B \cong C$.

Proof. This follows from Theorem 1.2 and results of Bass and Evans. See [4, Th. 2].

By Theorem 1.2, if $N$ is a non-zero uniserial module and $\operatorname{End}(N)$ is its endomorphism ring, then either $\operatorname{End}(N) / J(\operatorname{End}(N))$ is a division ring (that is, $\operatorname{End}(N)$ is a local ring) or $\operatorname{End}(N) / J(\operatorname{End}(N))$ is the direct product of two division rings. We shall say that a non-zero uniserial module is of type 1 if its endomorphism ring is local, and of type 2 otherwise. Hence a non-zero uniserial module $N$ is of type $d$ if and only if $\operatorname{End}(N) / J(\operatorname{End}(N))$ is the direct product of $d$ division rings (and only $d=1$ or $d=2$ can occur).

For instance, every commutative valuation ring is a uniserial module of type 1 as a module over itself. Further examples of uniserial modules of type 1 and examples of uniserial modules of type 2 will be given in Section 2 .

Lemma 1.4. Let $A, B$ be non-zero uniserial modules over an arbitrary ring $R$.

(a) If $f, g: A \rightarrow B$ are two homomorphisms, $f$ is injective and non-surjective, and $g$ is surjective and non-injective, then $f+g$ is an isomorphism.

(b) Conversely, suppose that $f_{1}, \ldots, f_{n}: A \rightarrow B$ are $n$ homomorphisms none of which is an isomorphism. If $f_{1}+\cdots+f_{n}$ is an isomorphism, then there 
exist two distinct indices $i, j=1,2, \ldots, n$ such that $f_{i}$ is injective and nonsurjective, and $f_{j}$ is surjective and non-injective.

Proof. The proof of (a) is elementary. For the proof of (b), consider the $n$ elements $\left(f_{1}+\cdots+f_{n}\right)^{-1} f_{i}$ of $\operatorname{End}\left(A_{R}\right)$. Their sum is $1_{A}$ and none of them is invertible in $\operatorname{End}\left(A_{R}\right)$. Hence $\operatorname{End}\left(A_{R}\right)$ is not a local ring. By Theorem 1.2 the ring $\operatorname{End}\left(A_{R}\right) / J\left(\operatorname{End}\left(A_{R}\right)\right)$ is canonically isomorphic to the direct product of two division rings $\operatorname{End}\left(A_{R}\right) / I$ and $\operatorname{End}\left(A_{R}\right) / J$. Now the conclusion follows easily.

The next proposition reduces the study of the Krull-Schmidt property for serial modules to the case of a direct sum of two uniserial modules. Its proof was inspired by the proof of [6, Lemma V.5.2].

Proposition 1.5. Suppose $A \oplus B=C_{1} \oplus \cdots \oplus C_{n}$, with $n \geq 2$ and $A$ uniserial. Then there are two distinct indices $i$ and $j$ and a direct decomposition $A^{\prime} \oplus B^{\prime}=C_{i} \oplus C_{j}$ of $C_{i} \oplus C_{j}$ such that $A \cong A^{\prime}$ and $B \cong B^{\prime} \oplus\left(\bigoplus_{k \neq i, j} C_{k}\right)$.

Proof. If $A=0$ the statement is trivial. Hence we can suppose $A \neq 0$. If the endomorphism ring $E=\operatorname{End}(A)$ of $A$ is local the proposition follows immediately from [6, Lemma V.5.2]. Hence we can suppose that the endomorphism ring $E$ has exactly two maximal ideals $I$ and $J$. Let $\iota_{A}, \pi_{A}, \iota_{B}, \pi_{B}$ and $\iota_{i}, \pi_{i}(i=1,2, \ldots, n)$ denote the injections and projections associated to the two direct decompositions $A \oplus B$ and $C_{1} \oplus \cdots \oplus C_{n}$. In $E$ we have

$$
1=\pi_{A} \iota_{A}=\pi_{A}\left(\sum_{i} \iota_{i} \pi_{i}\right) \iota_{A}=\sum_{i} \pi_{A} \iota_{i} \pi_{i} \iota_{A} .
$$

If one of the terms in this sum is invertible in $E, \pi_{A} \iota_{i} \pi_{i} \iota_{A}$ is invertible in $E$ say, then the composite mapping of $\pi_{i} \iota_{A}: A \rightarrow C_{i}$ and $\left(\pi_{A} \iota_{i} \pi_{i} \iota_{A}\right)^{-1} \pi_{A} \iota_{i}: C_{i} \rightarrow A$ is the identity mapping of $A$, so that $A$ is isomorphic to a non-zero direct summand $A^{\prime}$ of $C_{i}$. In this case we can take any index $j \neq i$ and we're done. Therefore we can suppose that none of the terms $\pi_{A} \iota_{i} \pi_{i} \iota_{A}$ is invertible in $E$. Then there exist two indices $i$ and $j$ such that $\pi_{A} \iota_{i} \pi_{i} \iota_{A} \in I \backslash J$ and $\pi_{A} \iota_{j} \pi_{j} \iota_{A} \in J \backslash I$ by Lemma 1.4(b). Then $\alpha=\pi_{A} \iota_{i} \pi_{i} \iota_{A}+\pi_{A} \iota_{j} \pi_{j} \iota_{A}$ is invertible in $E$ by Lemma 1.4(a), that is, $\alpha$ is an automorphism of $A$. Let $\iota^{\prime}: C_{i} \oplus C_{j} \rightarrow A \oplus B$ and $\pi^{\prime}: A \oplus B \rightarrow C_{i} \oplus C_{j}$ denote the injection and the projection associated to the direct summand $C_{i} \oplus C_{j}$ of $A \oplus B=C_{1} \oplus \cdots \oplus C_{n}$, so that $\alpha=\pi_{A} \iota^{\prime} \pi^{\prime} \iota_{A}$. Set $\gamma=\alpha^{-1} \pi_{A} \iota^{\prime}: C_{i} \oplus C_{j} \rightarrow A$. Then $\gamma \pi^{\prime} \iota_{A}=\alpha^{-1} \pi_{A} \iota^{\prime} \pi^{\prime} \iota_{A}=\alpha^{-1} \alpha=1_{A}$, and therefore $\gamma$ is a splitting epimorphism, that is, $A^{\prime} \oplus B^{\prime}=C_{i} \oplus C_{j}$, where $A^{\prime}=\pi^{\prime} \iota_{A}(A) \cong A$ and $B^{\prime}=\operatorname{ker} \gamma$.

Let $A$ and $B$ be two modules. We shall say that $A$ and $B$ belong to the same monogeny class if there exist a monomorphism $A \rightarrow B$ and a monomorphism $B \rightarrow A$. Similarly, we shall say that $A$ and $B$ belong to the same epigeny class if there exist an epimorphism $A \rightarrow B$ and an epimorphism $B \rightarrow A$. Clearly, these are two equivalence relations. Let $[A]_{m}$ and $[A]_{e}$ denote the monogeny class and the epigeny class of a module $A$.

The relationship between isomorphism, monogeny and epigeny classes is described in the next proposition.

Proposition 1.6. Let $A$ and $B$ be uniserial modules. Then $A \cong B$ if and only if $[A]_{m}=[B]_{m}$ and $[A]_{e}=[B]_{e}$. 
Proof. The "only if" implication is obvious. Conversely, suppose $[A]_{m}=[B]_{m}$ and $[A]_{e}=[B]_{e}$. Then there exist a monomorphism $A \rightarrow B$ and an epimorphism $A \rightarrow B$. If one of these two homomorphisms is an isomorphism, then $A \cong B$. If both these homomorphisms are not isomorphisms, then their sum is an isomorphism by Lemma 1.4(a).

Proposition 1.7. Let $A, U_{1}, \ldots, U_{n}$ be uniserial modules, $n \geq 2$ and $A \neq 0$. Suppose that $A$ is isomorphic to a direct summand of $U_{1} \oplus \cdots \oplus U_{n}$ and $A \neq U_{i}$ for every $i$. Then there are two distinct indices $i, j=1,2, \ldots, n$ such that $[A]_{m}=\left[U_{i}\right]_{m}$ and $[A]_{e}=\left[U_{j}\right]_{e}$.

Conversely, let $A, U, V$ be uniserial modules such that $[A]_{m}=[U]_{m}$ and $[A]_{e}=$ $[V]_{e}$. Then $A \oplus X \cong U \oplus V$ for some module $X$, necessarily uniserial, that is unique up to isomorphism.

Proof. Let $A, U_{1}, \ldots, U_{n}$ be uniserial modules, $n \geq 2$. Suppose that $A$ is isomorphic to a non-zero direct summand of $U_{1} \oplus \cdots \oplus U_{n}$ and that $A \neq U_{i}$ for every $i=1,2, \ldots, n$. By Proposition 1.5 there are two distinct indices $i$ and $j$ such that $A$ is isomorphic to a direct summand of $U_{i} \oplus U_{j}$. Hence there are two morphisms $A \rightarrow U_{i} \oplus U_{j}$ and $U_{i} \oplus U_{j} \rightarrow A$ whose composition is the identity morphism $1_{A}$ of $A$. It follows that there are four morphisms $f: A \rightarrow U_{i}, g: A \rightarrow U_{j}, h: U_{i} \rightarrow A$, $\ell: U_{j} \rightarrow A$ such that $h f+\ell g=1_{A}$. If $h f$ is an isomorphism, then $h$ and $f$ are isomorphisms by Lemma 1.1, and this is impossible because $A$ is not isomorphic to $U_{i}$. Hence $h f$ is not an isomorphism. Similarly $\ell g$ is not an isomorphism. If $E=\operatorname{End}(A)$ is a local ring, then the sum of two morphisms that are not invertible is not invertible. Since $h f+\ell g=1_{A}, E$ cannot be a local ring. By Theorem $1.2 E / J(E)$ is the direct product of the two division rings $E / I$ and $E / J$. From $h f+\ell g=1_{A}$ and the fact that $h f$ and $\ell g$ are not invertible in $E$ it follows that either $h f \in I \backslash J$ and $\ell g \in J \backslash I$ or $h f \in J \backslash I$ and $\ell g \in I \backslash J$. By symmetry we can suppose that $h f \notin I$ and $\ell g \notin J$, that is, $h f$ is a monomorphism and $\ell g$ is an epimorphism. By Lemma $1.1 f: A \rightarrow U_{i}$ and $h: U_{i} \rightarrow A$ are monomorphisms, and $g: A \rightarrow U_{j}$ and $\ell: U_{j} \rightarrow A$ are epimorphisms.

Conversely, let $A, U, V$ be uniserial modules such that $[A]_{m}=[U]_{m}$ and $[A]_{e}=$ $[V]_{e}$, so that there exist two monomorphisms $\alpha_{1}: A \rightarrow U$ and $\alpha_{2}: U \rightarrow A$ and two epimorphisms $\beta_{1}: A \rightarrow V$ and $\beta_{2}: V \rightarrow A$. If $A=0$, then $U=0$ and $V=0$, and the statement is trivial. Hence we can suppose $A \neq 0$, so that $U \neq 0$ and $V \neq 0$ also. Consider the homomorphisms

$$
\left(\begin{array}{c}
\alpha_{1} \\
\beta_{1}
\end{array}\right): A \rightarrow U \oplus V
$$

and

$$
\left(\begin{array}{ll}
\alpha_{2} & \beta_{2}
\end{array}\right): U \oplus V \rightarrow A,
$$

whose composite mapping is

$$
\left(\begin{array}{ll}
\alpha_{2} & \beta_{2}
\end{array}\right)\left(\begin{array}{c}
\alpha_{1} \\
\beta_{1}
\end{array}\right)=\alpha_{2} \alpha_{1}+\beta_{2} \beta_{1}: A \rightarrow A .
$$

If $\alpha_{2} \alpha_{1}: A \rightarrow A$ is an isomorphism, then both $\alpha_{1}$ and $\alpha_{2}$ are isomorphisms by Lemma 1.1, so that $A \oplus V \cong U \oplus V$. Hence in this case $X=V$ has the property required in the second part of the statement. Similarly, if $\beta_{2} \beta_{1}: A \rightarrow A$ is an isomorphism, then $A \oplus U \cong V \oplus U$ and $X=U$ has the required property. Hence we 
can suppose that neither $\alpha_{2} \alpha_{1}$ nor $\beta_{2} \beta_{1}$ are isomorphisms. Then $\gamma=\alpha_{2} \alpha_{1}+\beta_{2} \beta_{1}$ is an isomorphism by Lemma 1.4(a). The composite mapping

$$
\left(\gamma^{-1}\left(\begin{array}{cc}
\alpha_{2} & \beta_{2}
\end{array}\right)\right) \circ\left(\begin{array}{c}
\alpha_{1} \\
\beta_{1}
\end{array}\right): A \rightarrow U \oplus V \rightarrow A
$$

is the identity mapping of $A$. Hence

$$
U \oplus V \cong A \oplus \operatorname{ker}\left(\gamma^{-1}\left(\begin{array}{ll}
\alpha_{2} & \beta_{2}
\end{array}\right)\right)=A \oplus \operatorname{ker}\left(\begin{array}{ll}
\alpha_{2} & \beta_{2}
\end{array}\right) .
$$

Moreover

$$
\begin{aligned}
& \operatorname{ker}\left(\begin{array}{ll}
\alpha_{2} & \beta_{2}
\end{array}\right)=\left\{(u, v) \in U \oplus V \mid \alpha_{2}(u)+\beta_{2}(v)=0\right\} \\
& \quad=\left\{\left(\alpha_{2}^{-1}\left(\beta_{2}(-v)\right), v\right) \mid v \in V, \beta_{2}(v) \in \alpha_{2}(U)\right\} \cong \beta_{2}^{-1}\left(\alpha_{2}(U)\right),
\end{aligned}
$$

and $\beta_{2}^{-1}\left(\alpha_{2}(U)\right)$ is a uniserial module because it is a submodule of $V$. Hence $X=\operatorname{ker}\left(\begin{array}{lll}\alpha_{2} & \beta_{2}\end{array}\right)$ has the required property.

Finally, if $X^{\prime}$ is another module with $A \oplus X^{\prime} \cong U \oplus V$, then $A \oplus X^{\prime} \cong A \oplus X$, so that $X^{\prime} \cong X$ by Corollary 1.3 .

The next lemma is a further step in the proof of our main theorem (Theorem $1.9)$.

Lemma 1.8. Let $U_{1}, U_{2}, V_{1}, V_{2}$ be non-zero uniserial modules and suppose that $U_{1} \oplus U_{2} \cong V_{1} \oplus V_{2}$. Then $\left\{\left[U_{1}\right]_{m},\left[U_{2}\right]_{m}\right\}=\left\{\left[V_{1}\right]_{m},\left[V_{2}\right]_{m}\right\}$ and $\left\{\left[U_{1}\right]_{e},\left[U_{2}\right]_{e}\right\}=$ $\left\{\left[V_{1}\right]_{e},\left[V_{2}\right]_{e}\right\}$.

Proof. By symmetry, it is sufficient to prove that $\left\{\left[U_{1}\right]_{m},\left[U_{2}\right]_{m}\right\} \subseteq\left\{\left[V_{1}\right]_{m},\left[V_{2}\right]_{m}\right\}$ and $\left\{\left[U_{1}\right]_{e},\left[U_{2}\right]_{e}\right\} \subseteq\left\{\left[V_{1}\right]_{e},\left[V_{2}\right]_{e}\right\}$, and for this we must show that for every $U_{k}$, $k=1,2$, there exists an index $i$ such that $\left[U_{k}\right]_{m}=\left[V_{i}\right]_{m}$ and there exists an index $j$ such that $\left[U_{k}\right]_{e}=\left[V_{j}\right]_{e}$. This is obvious if $U_{k}$ is isomorphic to $V_{1}$ or $V_{2}$, and is shown in Proposition 1.7 if $U_{k}$ is not isomorphic to $V_{1}$ and $V_{2}$.

We are ready to prove our weak form of the Krull-Schmidt Theorem for serial modules. For the proof, define the "m-e collection" of a finite family of uniserial modules to be the collection of monogeny classes of its terms, each monogeny class being counted as often as it occurs, together with the collection of epigeny classes of the terms, again counting multiplicity.

Theorem 1.9. Let $U_{1}, \ldots, U_{n}, V_{1}, \ldots, V_{t}$ be non-zero uniserial modules. Then $U_{1} \oplus$ $\cdots \oplus U_{n} \cong V_{1} \oplus \cdots \oplus V_{t}$ if and only if $n=t$ and there are two permutations $\sigma, \tau$ of $\{1,2, \ldots, n\}$ such that $\left[U_{\sigma(i)}\right]_{m}=\left[V_{i}\right]_{m}$ and $\left[U_{\tau(i)}\right]_{e}=\left[V_{i}\right]_{e}$ for every $i=1,2, \ldots, n$.

Note that in the terminology just introduced the theorem can be restated: "The direct sums of two finite families of uniserial modules are isomorphic if and only if the two families have the same m-e collections."

Proof. $(\Rightarrow)$ We have already remarked in the introduction that $U_{1} \oplus \cdots \oplus U_{n} \cong$ $V_{1} \oplus \cdots \oplus V_{t}$ implies $n=t$ because $n$ and $t$ are the Goldie dimensions of $U_{1} \oplus \cdots \oplus U_{n}$ and $V_{1} \oplus \cdots \oplus V_{t}$ respectively.

Suppose, first, that no $V_{i}$ is isomorphic to $U_{1}$. Then, by Proposition 1.7, we can renumber the $V_{i}^{\prime}$ 's so that $\left[U_{1}\right]_{m}=\left[V_{1}\right]_{m}$ and $\left[U_{1}\right]_{e}=\left[V_{2}\right]_{e}$. Moreover, again by Proposition 1.7, there is a uniserial module $X$ such that $U_{1} \oplus X \cong V_{1} \oplus V_{2}$. We now have the following three decompositions of $M$ :

$$
U_{1} \oplus U_{2} \oplus \cdots \oplus U_{n} \cong V_{1} \oplus V_{2} \oplus \cdots \oplus V_{n} \cong U_{1} \oplus X \oplus V_{3} \oplus \cdots \oplus V_{n} .
$$


Since uniserial modules cancel from direct sums, we get that $U_{2} \oplus \cdots \oplus U_{n} \cong$ $X \oplus V_{3} \oplus \cdots \oplus V_{n}$, and thus induction shows that $\left\{U_{2}, \ldots, U_{n}\right\}$ and $\left\{X, V_{3}, \ldots, V_{n}\right\}$ have the same m-e collections. Therefore the first and third decompositions in (1) have the same m-e collections. Since $U_{1} \oplus X \cong V_{1} \oplus V_{2}$, Lemma 1.8 shows that the second and the third decompositions in (1) have the same m-e collections. Hence the first and second decompositions have the same m-e collections, as desired.

Suppose, on the other hand, that some $V_{i}$ is isomorphic to $U_{1}$. After renumbering we can assume that $U_{1} \cong V_{1}$. Then, since uniserial modules cancel from direct sums, we are once again done by induction.

$(\Leftarrow)$ Here we assume that the families $\left\{U_{1}, \ldots, U_{n}\right\}$ and $\left\{V_{1}, \ldots, V_{n}\right\}$ have the same m-e collections and we want to show that their direct sums are isomorphic. Thus we have $\left[U_{1}\right]_{m}=\left[V_{i}\right]_{m}$ and $\left[U_{1}\right]_{e}=\left[V_{j}\right]_{e}$ for some $i, j$.

Suppose first that $i \neq j$. Then we can renumber the $V_{i}$ 's so that $\left[U_{1}\right]_{m}=$ $\left[V_{1}\right]_{m}$ and $\left[U_{1}\right]_{e}=\left[V_{2}\right]_{e}$. By Proposition 1.7 we have $U_{1} \oplus X \cong V_{1} \oplus V_{2}$ for some uniserial $X$; and by Lemma 1.8 the families $\left\{U_{1}, X\right\}$ and $\left\{V_{1}, V_{2}\right\}$ have the same m-e collections. It follows that $\left\{U_{1}, X, V_{3}, \ldots, V_{n}\right\}$ and $\left\{V_{1}, V_{2}, V_{3}, \ldots, V_{n}\right\}$ have the same m-e collections. Then $\left\{U_{1}, X, V_{3}, \ldots, V_{n}\right\}$ and $\left\{U_{1}, \ldots, U_{n}\right\}$ have the same m-e collections, so that $\left\{X, V_{3}, \ldots, V_{n}\right\}$ and $\left\{U_{2}, \ldots, U_{n}\right\}$ have the same m-e collections. By induction $X \oplus V_{3} \oplus \cdots \oplus V_{n} \cong U_{2} \oplus \cdots \oplus U_{n}$, so that $V_{1} \oplus V_{2} \oplus \cdots \oplus V_{n} \cong$ $U_{1} \oplus X \oplus V_{3} \oplus \cdots \oplus V_{n} \cong U_{1} \oplus U_{2} \oplus \cdots \oplus U_{n}$, as desired.

Suppose, on the other hand, that $i=j$. We may assume, then, that $i=1$, so that $U_{1} \cong V_{1}$ by Proposition 1.6. By induction $V_{2} \oplus \cdots \oplus V_{n} \cong U_{2} \oplus \cdots \oplus U_{n}$, and thus $V_{1} \oplus \cdots \oplus V_{n} \cong U_{1} \oplus \cdots \oplus U_{n}$.

Theorem 1.9 is the "best" uniqueness result that holds for serial modules, in the sense that, as we shall show in Example 2.1, given two arbitrary permutations of $\{1,2, \ldots, n\}$, there are a serial module $M$ of Goldie dimension $n$ over a suitable ring $R$ and a pair of decompositions of $M$ with those two permutations of the monogeny classes and the epigeny classes. Hence the isomorphism classes of the direct summands in a decomposition of a serial module as a finite direct sum of non-zero uniserial modules do depend on the decomposition, but the monogeny classes and the epigeny classes of the uniserial direct summands in a decomposition do not depend on the decomposition itself. Moreover, the isomorphism class of a uniserial module $N$ is completely determined by its monogeny class and its epigeny class (Proposition 1.6).

Definition 1.10. We shall say that a non-zero uniserial module $A$ is a KrullSchmidt module if either

(a) for every submodule $A^{\prime} \subseteq A^{\prime \prime} \subseteq A, A^{\prime} \cong A$ implies $A^{\prime \prime} \cong A$, or

(b) for every submodule $B^{\prime} \subseteq B \subseteq A, A / B \cong A$ implies $A / B^{\prime} \cong A$.

Thus a non-zero uniserial module $A$ is a Krull-Schmidt module if and only if either for every module $B,[A]_{m}=[B]_{m}$ implies $A \cong B$, or for every module $B$, $[A]_{e}=[B]_{e}$ implies $A \cong B$. In other words, we call a non-zero uniserial module a Krull-Schmidt module if (up to isomorphism) it is the only module in its monogeny class or the only module in its epigeny class. The reason for this terminology is:

Corollary 1.11. A non-zero uniserial module $A$ is a Krull-Schmidt module if and only if whenever $U_{1}, \ldots, U_{n}$ are uniserial modules and $A$ is isomorphic to a direct summand of $U_{1} \oplus \cdots \oplus U_{n}$, then $A$ must be isomorphic to $U_{i}$ for some $i=1,2, \ldots, n$. 
Proof. Suppose that $A$ is a uniserial Krull-Schmidt module and $U_{1}, \ldots, U_{n}$ are uniserial modules such that $A$ is isomorphic to a direct summand of $U_{1} \oplus \cdots \oplus U_{n}$. By Proposition 1.7 the monogeny and epigeny classes of $A$ must appear in the m-e collection of $\left\{U_{1}, \ldots, U_{n}\right\}$. Since $A$ is the unique module in its monogeny class, or epigeny class, we must have $A$ isomorphic to some $U_{i}$ as claimed.

Conversely, suppose that $A$ is a non-zero uniserial module that is not a KrullSchmidt module. Then there exist a uniserial module $U_{1} \neq A$ with $[A]_{m}=\left[U_{1}\right]_{m}$ and a uniserial module $U_{2} \neq A$ with $[A]_{e}=\left[U_{2}\right]_{e}$. Hence $A$ is a direct summand of $U_{1} \oplus U_{2}$ by Proposition 1.7.

From [6, Lemma V.5.2] and Corollary 1.11 we obtain

Proposition 1.12. Every uniserial module of type 1 is a Krull-Schmidt module.

As an application of Theorem 1.9 we shall compute an upper bound for the number of non-isomorphic uniserial direct summands of a serial module of finite Goldie dimension.

Corollary 1.13. Let $U_{1}, \ldots, U_{n}$ be Krull-Schmidt uniserial modules, $V_{1}, \ldots, V_{r}$ non-Krull-Schmidt uniserial modules, $t_{1}, \ldots, t_{n}, u_{1}, \ldots, u_{r}$ non-negative integers, $M=U_{1}^{t_{1}} \oplus \cdots \oplus U_{n}^{t_{n}} \oplus V_{1}^{u_{1}} \oplus \cdots \oplus V_{r}^{u_{r}}$. Then $M$ has at most $n+r^{2}$ non-isomorphic uniserial direct summands $\neq 0$.

Proof. Let $A$ be a non-zero uniserial direct summand of $M$. Then by Proposition 1.7 the monogeny and epigeny classes of $A$ appear in the m-e collection of $A$. Say $[A]_{m}=[X]_{m}$ and $[A]_{e}=[Y]_{e}$, where $X$ is some $U_{i}$ or $V_{j}$ and the same is true of $Y$. We consider two cases.

Case 1. $A$ is a Krull-Schmidt module. Then $A$ is isomorphic to some $U_{i}$ or $V_{j}$. Since $A$ is a Krull-Schmidt module, we therefore have $A \cong U_{i}$. We conclude that there are at most $n$ possibilities for $A$.

Case 2. $A$ is not a Krull-Schmidt module. Then $X$ and $Y$ are among the $V_{j}$ 's. Thus there are at most $r$ possibilities for each of the monogeny and epigeny classes of $A$. Hence, by Proposition 1.6, there are at most $r^{2}$ possibilities for the isomorphism class of $A$.

\section{EXAMPLES}

Now we show that Krull-Schmidt fails for finitely presented modules over serial rings. This answers Warfield's question in the negative (see the introduction). Our first example is partially based on a construction due to Luigi Salce and the author [3, p. 502]

Example 2.1. Let $n \geq 2$ be an integer. Then there exist a serial ring $R$ and $n^{2}$ pairwise non-isomorphic finitely presented uniserial $R$-modules $U_{i, j}, i, j=1, \ldots, n$, with the following properties:

(a) $\left[U_{i, j}\right]_{m}=\left[U_{k, \ell}\right]_{m}$ if and only if $i=k$;

(b) $\left[U_{i, j}\right]_{e}=\left[U_{k, \ell}\right]_{e}$ if and only if $j=\ell$.

In particular, if $\sigma, \tau$ are two permutations of $\{1,2, \ldots, n\}$, then

$$
U_{1,1} \oplus U_{2,2} \oplus \cdots \oplus U_{n, n} \cong U_{\sigma(1), \tau(1)} \oplus U_{\sigma(2), \tau(2)} \oplus \cdots \oplus U_{\sigma(n), \tau(n)} .
$$


Proof. Let $\mathbf{Q}$ be the field of rational numbers, $\mathbf{Z}$ the ring of integers, $p$ and $q$ distinct primes, $\mathbf{Z}_{p}$ and $\mathbf{Z}_{q}$ the localizations of $\mathbf{Z}$ at the two distinct maximal ideals $(p)$ and $(q)$ respectively, and $M_{n}(\mathbf{Q})$ the ring of all $n \times n$-matrices over $\mathbf{Q}$. Let

$$
\Lambda_{p}=\left(\begin{array}{cccc}
\mathbf{Z}_{p} & \mathbf{Z}_{p} & \ldots & \mathbf{Z}_{p} \\
p \mathbf{Z}_{p} & \mathbf{Z}_{p} & \ldots & \mathbf{Z}_{p} \\
\vdots & & \ddots & \\
p \mathbf{Z}_{p} & p \mathbf{Z}_{p} & \ldots & \mathbf{Z}_{p}
\end{array}\right)
$$

and

$$
\Lambda_{q}=\left(\begin{array}{cccc}
\mathbf{Z}_{q} & \mathbf{Z}_{q} & \ldots & \mathbf{Z}_{q} \\
q \mathbf{Z}_{q} & \mathbf{Z}_{q} & \ldots & \mathbf{Z}_{q} \\
\vdots & & \ddots & \\
q \mathbf{Z}_{q} & q \mathbf{Z}_{q} & \ldots & \mathbf{Z}_{q}
\end{array}\right)
$$

be the subrings of $M_{n}(\mathbf{Q})$ whose elements on and above the diagonal are in $\mathbf{Z}_{p}$ (resp. in $\mathbf{Z}_{q}$ ) and whose elements under the diagonal are in $p \mathbf{Z}_{p}$ (resp. in $q \mathbf{Z}_{q}$ ). Set

$$
R=\left(\begin{array}{cc}
\Lambda_{p} & 0 \\
M_{n}(\mathbf{Q}) & \Lambda_{q}
\end{array}\right)
$$

so that $R$ is a subring of the $\operatorname{ring} M_{2 n}(\mathbf{Q})$ of $2 n \times 2 n$-matrices.

For any ring $S$ let $U(S)$ denote the group of units of $S$. It is easily seen that

$$
U\left(\Lambda_{p}\right)=\left(\begin{array}{cccc}
U\left(\mathbf{Z}_{p}\right) & \mathbf{Z}_{p} & \ldots & \mathbf{Z}_{p} \\
p \mathbf{Z}_{p} & U\left(\mathbf{Z}_{p}\right) & \ldots & \mathbf{Z}_{p} \\
\vdots & & \ddots & \\
p \mathbf{Z}_{p} & p \mathbf{Z}_{p} & \ldots & U\left(\mathbf{Z}_{p}\right)
\end{array}\right)
$$

and

$$
U(R)=\left(\begin{array}{cc}
U\left(\Lambda_{p}\right) & 0 \\
M_{n}(\mathbf{Q}) & U\left(\Lambda_{q}\right)
\end{array}\right),
$$

so that the Jacobson radicals of these rings are

$$
J\left(\Lambda_{p}\right)=\left(\begin{array}{cccc}
p \mathbf{Z}_{p} & \mathbf{Z}_{p} & \cdots & \mathbf{Z}_{p} \\
p \mathbf{Z}_{p} & p \mathbf{Z}_{p} & \cdots & \mathbf{Z}_{p} \\
\vdots & & \ddots & \\
p \mathbf{Z}_{p} & p \mathbf{Z}_{p} & \cdots & p \mathbf{Z}_{p}
\end{array}\right)
$$

and

$$
J(R)=\left(\begin{array}{cc}
J\left(\Lambda_{p}\right) & 0 \\
M_{n}(\mathbf{Q}) & J\left(\Lambda_{q}\right)
\end{array}\right)
$$

Let $e_{i}=e_{i i} \in R, i=1,2, \ldots, 2 n$, be the $(i, i)$ matrix units of the ring $M_{2 n}(\mathbf{Q})$. Easy calculations show that the $R$-modules $R e_{i}$ and $e_{i} R$ are uniserial, so that $R$ is a serial ring with $2 n$ simple pairwise non-isomorphic right modules $e_{i} R / e_{i} J(R)$, $i=1,2, \ldots, 2 n$. 
For instance, consider the right ideal

$$
e_{n+1} R=\left(\begin{array}{cccccc}
0 & \ldots & 0 & 0 & \ldots & 0 \\
\vdots & & & & & \vdots \\
0 & \ldots & 0 & 0 & \ldots & 0 \\
\mathbf{Q} & \ldots & \mathbf{Q} & \mathbf{Z}_{q} & \ldots & \mathbf{Z}_{q} \\
\vdots & & & & & \vdots \\
0 & \ldots & 0 & 0 & \ldots & 0
\end{array}\right) .
$$

This right ideal of $R$ is isomorphic to the right $R$-module

$$
V=(\underbrace{\mathbf{Q}, \ldots, \mathbf{Q}}_{n}, \underbrace{\mathbf{Z}_{q}, \ldots, \mathbf{Z}_{q}}_{n})
$$

of $1 \times 2 n$-matrices, where the $R$-module structure on $V$ is given by matrix multiplication (the elements of $V$ are $1 \times 2 n$-matrices and the elements of the ring $R$ are $2 n \times 2 n$-matrices). Set

$$
W=(\underbrace{\mathbf{Q}, \ldots, \mathbf{Q}}_{n}, \underbrace{0, \ldots, 0}_{n})
$$

for every $j=1,2, \ldots, n$ set

$$
V_{j}=(\underbrace{\mathbf{Q}, \ldots, \mathbf{Q}}_{n}, \underbrace{q \mathbf{Z}_{q}, \ldots, q \mathbf{Z}_{q}}_{j-1}, \underbrace{\mathbf{Z}_{q}, \ldots, \mathbf{Z}_{q}}_{n-j+1})
$$

and for every $i=1,2, \ldots, n$ set

$$
X_{i}=(\underbrace{p \mathbf{Z}_{p}, \ldots, p \mathbf{Z}_{p}}_{i-1}, \underbrace{\mathbf{Z}_{p}, \ldots, \mathbf{Z}_{p}}_{n-i+1}, \underbrace{0, \ldots, 0}_{n}) .
$$

It is easily seen that $X_{i} J(R)=X_{i+1}$ for every $i=1,2, \ldots, n-1, X_{n} J(R)=p X_{1}$, $V_{j} J(R)=V_{j+1}$ for every $j=1,2, \ldots, n-1$, and $V_{n} J(R)=q V_{1}$. Hence the unique (infinite) composition series of $V$ is

$$
\begin{aligned}
V= & V_{1} \supset V_{2} \supset V_{3} \supset \cdots \supset V_{n} \\
& \supset q V_{1} \supset q V_{2} \supset q V_{3} \supset \cdots \supset q V_{n} \\
& \supset q^{2} V_{1} \supset q^{2} V_{2} \supset q^{2} V_{3} \supset \cdots \supset q^{2} V_{n} \\
& \supset \cdots \supset W \supset \cdots \\
& \supset p^{-1} X_{1} \supset p^{-1} X_{2} \supset p^{-1} X_{3} \supset \cdots \supset p^{-1} X_{n} \\
& \supset X_{1} \supset X_{2} \supset X_{3} \supset \cdots \supset X_{n} \\
& \supset p X_{1} \supset p X_{2} \supset p X_{3} \supset \cdots \supset p X_{n} \\
& \supset \cdots \supset 0 .
\end{aligned}
$$

Note that

$$
\begin{gathered}
X_{i} / X_{i+1} \cong e_{i} R / e_{i} J(R) \quad \text { for every } i=1,2, \ldots, n-1, \\
X_{n} / p X_{1} \cong e_{n} R / e_{n} J(R), \\
V_{j} / V_{j+1} \cong e_{n+j} R / e_{n+j} J(R) \text { for every } j=1,2, \ldots, n-1, \\
V_{n} / q V_{1} \cong e_{2 n} R / e_{2 n} J(R) .
\end{gathered}
$$

We now show that the $n^{2} R$-modules $U_{i, j}=V_{j} / X_{i}, i, j=1,2, \ldots, n$, have the required properties. 
(a) $(\Rightarrow)$ Suppose that $\left[U_{i, j}\right]_{m}=\left[U_{k, \ell}\right]_{m}$ for some $i, j, k, \ell$. Then the socle $\operatorname{Soc}\left(U_{i, j}\right)$ of $U_{i, j}$ and the $\operatorname{socle} \operatorname{Soc}\left(U_{k, \ell}\right)$ of $U_{k, \ell}$ are isomorphic. But for every index $i, j$, the simple module $\operatorname{Soc}\left(U_{i, j}\right)=\operatorname{Soc}\left(V_{j} / X_{i}\right)$ is equal to $X_{i-1} / X_{i} \cong$ $e_{i-1} R / e_{i-1} J(R)$ if $i=2,3, \ldots, n$, and is equal to $p^{-1} X_{n} / X_{1} \cong e_{n} R / e_{n} J(R)$ if $i=1$. Therefore $\operatorname{Soc}\left(U_{i, j}\right) \cong \operatorname{Soc}\left(U_{k, \ell}\right)$ implies $i=k$.

$(\Leftarrow)$ We must show that $\left[U_{i, j}\right]_{m}=\left[U_{i, \ell}\right]_{m}$ for every index $i, j, \ell$. Without loss of generality we may suppose $j \leq \ell$, so that $U_{i, j} \supseteq U_{i, \ell}$. In particular there is a monomorphism $U_{i, \ell} \rightarrow U_{i, j}$. Conversely, multiplication by $q$ is an endomorphism of $V$ that maps the submodules $V_{j}$ of $V$ to $q V_{j}$, and maps the submodules $X_{i}$ to $X_{i}$. Therefore multiplication by $q$ induces an isomorphism between $V_{j} / X_{i}=U_{i, j}$ and $p V_{j} / X_{i} \subseteq V_{\ell} / X_{i}=U_{i, \ell}$. Therefore there is a monomorphism $U_{i, j} \rightarrow U_{i, \ell}$, and $\left[U_{i, j}\right]_{m}=\left[U_{i, \ell}\right]_{m}$.

(b) $(\Rightarrow)$ An easy computation shows that for every index $i, j$ we have $U_{i, j} / J\left(U_{i, j}\right)$ $\cong V_{j} / J\left(V_{j}\right) \cong e_{n+j} R / e_{n+j} J(R)$. Suppose that $\left[U_{i, j}\right]_{e}=\left[U_{k, \ell}\right]_{e}$ for some $i, j, k, \ell$. Then $U_{i, j} / J\left(U_{i, j}\right) \cong U_{k, \ell} / J\left(U_{k, \ell}\right)$, so that $e_{n+j} R / e_{n+j} J(R) \cong e_{n+\ell} R / e_{n+\ell} J(R)$, and thus $j=\ell$.

$(\Leftarrow)$ We must show that $\left[U_{i, j}\right]_{e}=\left[U_{k, j}\right]_{e}$ for every $i, j, k$. By symmetry we may suppose $i \leq k$, so that $X_{i} \supseteq X_{k}$. In particular there is a canonical epimorphism of $U_{k, j}=V_{j} / X_{k}$ onto $U_{i, j}=V_{j} / X_{i}$. Conversely, multiplication by $p$ is an endomorphism of $V$ that maps the submodules $V_{j}$ of $V$ to $V_{j}$, and maps the submodules $X_{i}$ to $p X_{i}$. Hence multiplication by $p$ induces an isomorphism between $V_{j} / X_{i}=U_{i, j}$ and $V_{j} / p X_{i}$. Since $p X_{i} \subseteq X_{k}$, there is an onto mapping $V_{j} / p X_{i} \rightarrow V_{j} / X_{k}=U_{k, j}$. This shows that there is an epimorphism $U_{i, j} \rightarrow U_{k, j}$, so that $\left[U_{i, j}\right]_{e}=\left[U_{k, j}\right]_{e}$. This completes the proof.

The module $M=U_{1,1} \oplus U_{2,2} \oplus \cdots \oplus U_{n, n}$ in Example 2.1 shows that given any two permutations $\sigma, \tau$ of $\{1,2, \ldots, n\}$, there is a pair of decompositions of $M$ with those $\sigma, \tau$ satisfying the conditions of Theorem 1.9. In particular $M$ has $n$ ! essentially different decompositions (essentially different in the sense of the KrullSchmidt Theorem), and $M$ has $n^{2}$ non-isomorphic uniserial direct summands $\neq 0$ (see Corollary 1.13).

Also note that the modules $U_{i, j}$ are examples of uniserial modules that are not Krull-Schmidt modules.

If $\sigma$ and $\tau$ are two permutations of $\{1,2, \ldots, n\}$ with $\sigma(i) \neq \tau(i)$ for every $i=1,2, \ldots, n$ and we set $U_{i}=U_{i, i}$ and $V_{i}=U_{\sigma(i), \tau(i)}$, where the $U_{i, j}$ are the modules of Example 2.1, we get

Example 2.2. Let $n \geq 2$ be an integer. There exists a serial ring $R$ with $2 n$ pairwise non-isomorphic finitely presented uniserial modules $U_{1}, U_{2}, \ldots, U_{n}, V_{1}$, $V_{2}, \ldots, V_{n}$ such that $U_{1} \oplus U_{2} \oplus \cdots \oplus U_{n} \cong V_{1} \oplus V_{2} \oplus \cdots \oplus V_{n}$.

Theorem 1.9, Example 2.1 and Example 2.2 answer Warfield's question completely.

Example 2.3. Let $U$ be a non-zero uniserial right $R$-module. Then $U$ is a module of type 1 if at least one of the following conditions holds:

(a) $U$ is projective;

(b) $U$ is injective;

(c) $U$ is artinian;

(d) $U$ is noetherian;

(e) $R$ is commutative; 
(f) $R$ is right noetherian.

Proof. (a). If $U$ is projective, every surjective endomorphism of $U$ splits. Since $U$ is indecomposable, every surjective endomorphism of $U$ is an automorphism. Hence $\operatorname{End}(U)$ is local.

(b). Every injective indecomposable module has a local endomorphism ring.

(c). If a uniserial module $U$ is artinian, its lattice of submodules is well ordered by inclusion, so that every injective endomorphism of $U$ is an automorphism. Hence $\operatorname{End}(U)$ is local.

(d). Dual to (c).

(e) and (f). If a uniserial module $U$ is of type 2, then it has a surjective noninjective endomorphism $f$ and an injective non-surjective endomorphism $g$. These endomorphisms induce two isomorphisms $\widetilde{f}: U / \operatorname{ker}(f) \rightarrow U$ and $\widetilde{g}: U \rightarrow g(U)$. It follows that $g(U) / g(\operatorname{ker}(f)) \cong U$, so that $U$ is a shrinkable module in the sense of [3], that is, it is isomorphic to a proper submodule of a proper quotient of itself. Now [3, Cor. 3$]$ yields the conclusion.

Example 2.4. There exist uniserial cyclic modules of type 2 that are KrullSchmidt modules.

Proof. We can apply Facchini and Salce's construction [3, p. 502] to $\mathbf{Z}_{p}, \mathbf{Z}_{q}$ and $\mathbf{Q}$ to get a ring

and a right ideal

$$
R=\left(\begin{array}{cc}
\mathbf{Z}_{p} & 0 \\
\mathbf{Q} & \mathbf{Z}_{q}
\end{array}\right)
$$

$$
H=\left(\begin{array}{ll}
\mathbf{Z}_{p} & 0 \\
\mathbf{Z}_{p} & 0
\end{array}\right)
$$

of $R$ such that $R / H$ is a right uniserial $R$-module (the right ideals of $R$ containing $H$ are exactly of the form

$$
\left(\begin{array}{cc}
\mathbf{Z}_{p} & 0 \\
J & 0
\end{array}\right) \quad \text { and } \quad\left(\begin{array}{cc}
\mathbf{Z}_{p} & 0 \\
\mathbf{Q} & I
\end{array}\right)
$$

where $J$ is a $\mathbf{Z}_{p}$-submodule of $\mathbf{Q}$ containing $\mathbf{Z}_{p}$ and $I$ is an ideal of $\mathbf{Z}_{q}$ ). The endomorphism ring of $R / H$ is isomorphic to $\mathbf{Z}_{p} \cap \mathbf{Z}_{q}$, which is not local. Therefore $R / H$ is a uniserial module of type 2 .

The submodules of $R / H$ isomorphic to $R / H$ are the modules

$$
\left(\begin{array}{cc}
\mathbf{Z}_{p} & 0 \\
\mathbf{Q} & q^{n} \mathbf{Z}_{q}
\end{array}\right) / H, \quad n \geq 0
$$

(they are isomorphic to $R / H$ via multiplication by $q^{n}$ ). Hence every submodule of $R / H$ that contains a submodule isomorphic to $R / H$ is isomorphic to $R / H$. Therefore $R / H$ is a Krull-Schmidt uniserial $R$-module.

\section{Miscellaneous minor Results}

3.1. The endomorphism ring of a serial module of finite Goldie dimension is semilocal. For a module $N$ let $\operatorname{dim}(N)$ and $\operatorname{codim}(N)$ denote the Goldie dimension and the dual Goldie dimension of $N$ respectively (see [4] or [7]). If $N$ is a non-zero uniserial module, then $\operatorname{dim}(N)=\operatorname{codim}(N)=1$. Since the Goldie dimension and the dual Goldie dimension are additive functions, that is, their value on a finite direct sum is the sum of their values on the summands, for a serial module 
$M$ of finite Goldie dimension $n$ one has $\operatorname{dim}(M)=\operatorname{codim}(M)=n$. From Theorem $3(3)$ of [4] it follows immediately that $\operatorname{End}(M)$ is a semilocal ring, that is, $\operatorname{End}(M)$ modulo its Jacobson radical is a semisemiple artinian ring. More precisely, by $[4$, Th. 3(3)] we have that the Goldie dimension of the $\operatorname{ring} \operatorname{End}(M) / J(\operatorname{End}(M))$ is $\leq 2 n$, i.e., the ring $\operatorname{End}(M) / J(\operatorname{End}(M))$, as a module over itself, is a direct sum of at most $2 n$ simple modules. This partially generalizes Theorem 1.2.

An immediate consequence of this fact is the $n$-th root uniqueness (see $[2$, Prop. 2.1]): if $A$ and $B$ are serial modules of finite Goldie dimension, $n$ is a positive integer and $A^{n} \cong B^{n}$, then $A \cong B$. But this also follows from Theorem 1.9.

Dolors Herbera and Nguyen Viet Dung (independently) have remarked that most results of this paper hold not only for uniserial modules, but also for arbitrary modules $N$ with $\operatorname{dim}(N)=\operatorname{codim}(N)=1$.

3.2. The Grothendieck group of serial modules of finite Goldie dimension is free. Given a class $\mathcal{C}$ of modules over a fixed ring $R$, if the class $\mathcal{C}$ is closed for finite direct sums and has just a set of isomorphism classes, it is possible to construct the Grothendieck group of $\mathcal{C}$. The situation is particularly good if $\mathcal{C}$ contains the zero module and the cancellation property holds in $\mathcal{C}$, because in that case the isomorphism classes form a commutative monoid with the cancellation property, and the smallest abelian group that contains it is the Grothendieck group of the class $\mathcal{C}$ considered. If the Krull-Schmidt Theorem holds in $\mathcal{C}$, the Grothendieck group is free, and in fact the structure of the Grothendieck group of $\mathcal{C}$ shows how far the behavior of the direct sum decompositions of the modules in the class is from uniqueness.

The class $\mathcal{S}_{R}$ of serial modules of finite Goldie dimension over a ring $R$ is closed for finite direct sums, and the cancellation property holds in $\mathcal{S}_{R}$ (Corollary 1.3). We have seen that Krull-Schmidt fails for serial modules, but nevertheless in this section we shall show that the Grothendieck group of $\mathcal{S}_{R}$ is free.

Let $R$ be a fixed associative ring with 1 . If $U_{1}, \ldots, U_{n}, V_{1}, \ldots, V_{t}$ are non-zero uniserial right $R$-modules, we shall say that the (external) direct sums $U_{1} \oplus \cdots \oplus U_{n}$ and $V_{1} \oplus \cdots \oplus V_{t}$ are equivalent, and write $U_{1} \oplus \cdots \oplus U_{n} \sim V_{1} \oplus \cdots \oplus V_{t}$, if $n=t$ and, after a reordering of the summands, $U_{i}$ is isomorphic to $V_{i}$ for every $i=1,2, \ldots, n$. We shall denote the equivalence class of $U_{1} \oplus \cdots \oplus U_{n}$ modulo $\sim$ by $\left[U_{1} \oplus \cdots \oplus U_{n}\right]_{\sim}$. The set $\mathcal{D}=\left\{\left[U_{1} \oplus \cdots \oplus U_{n}\right]_{\sim} \mid n \geq 0, U_{1}, \ldots, U_{n}\right.$ non-zero uniserial right $R$-modules $\}$ of all these equivalence classes is a commutative monoid with respect to the addition (induced by the external direct sum)

$$
\left[U_{1} \oplus \cdots \oplus U_{n}\right]_{\sim}+\left[V_{1} \oplus \cdots \oplus V_{t}\right]_{\sim}=\left[U_{1} \oplus \cdots \oplus U_{n} \oplus V_{1} \oplus \cdots \oplus V_{t}\right]_{\sim} .
$$

Similarly, the isomorphism classes $[M]$ of the modules in the class $\mathcal{S}_{R}$ of serial right $R$-modules of finite Goldie dimension form a commutative monoid $\operatorname{Groth}\left(\mathcal{S}_{R}\right)^{+}$ with the cancellation property with respect to the addition defined by

$$
[M]+[N]=[M \oplus N]
$$

for every $M, N \in \mathcal{S}_{R}$. Therefore this monoid is contained in a unique smallest abelian group, the Grothendieck group $\operatorname{Groth}\left(\mathcal{S}_{R}\right)$ of serial $R$-modules of finite Goldie dimension. There is a canonical monoid homomorphism $\omega: \mathcal{D} \rightarrow \operatorname{Groth}\left(\mathcal{S}_{R}\right)$ defined by $\omega\left(\left[U_{1} \oplus \cdots \oplus U_{n}\right]_{\sim}\right)=\left[U_{1} \oplus \cdots \oplus U_{n}\right]$ for every element $\left[U_{1} \oplus \cdots \oplus U_{n}\right]_{\sim}$ of $\mathcal{D}$.

Let $\mathcal{M}=\left\{[V]_{m} \mid V\right.$ a non-zero uniserial $R$-module $\}$ denote the set of all monogeny classes of non-zero uniserial right $R$-modules, and $\mathcal{E}=\left\{[V]_{e} \mid V\right.$ a 
non-zero uniserial $R$-module $\}$ the set of all epigeny classes of non-zero uniserial right $R$-modules. Let $\mathbf{F M}_{\mathcal{M} \times \mathcal{E}}(\mathbf{Z})$ be the free abelian group of all the $\mathcal{M} \times \mathcal{E}$ matrices with entries in $\mathbf{Z}$ and at most finitely many non-zero entries. For every non-zero uniserial right $R$-module $V$ let $E_{V} \in \mathbf{F M}_{\mathcal{M} \times \mathcal{E}}(\mathbf{Z})$ denote the matrix whose entries are all zero except for the $\left([V]_{m},[V]_{e}\right)$ entry that is equal to 1 . Let $F$ be the additive submonoid of $\mathbf{F M}_{\mathcal{M} \times \mathcal{E}}(\mathbf{Z})$ generated by all the $E_{V}, V$ an arbitrary non-zero uniserial right $R$-module. The $E_{V}$ 's generate $F$ freely, so that $F$ is a free additive monoid. There is a monoid homomorphism $\varphi: \mathcal{D} \rightarrow \mathbf{F M}_{\mathcal{M} \times \mathcal{E}}(\mathbf{Z})$ defined by $\varphi\left(\left[U_{1} \oplus \cdots \oplus U_{n}\right]_{\sim}\right)=E_{U_{1}}+\cdots+E_{U_{n}}$ for every element $\left[U_{1} \oplus \cdots \oplus U_{n}\right]_{\sim}$ of $\mathcal{D}$. This mapping $\varphi$ is a monomorphism by Proposition 1.6, and its image is $F$. Hence $\mathcal{D} \cong F$ is a free commutative monoid.

Now let $\mathbf{Z}^{(\mathcal{M})}$ and $\mathbf{Z}^{(\mathcal{E})}$ be the abelian groups freely generated by the sets $\mathcal{M}$ and $\mathcal{E}$ respectively. Note that if $A \in \mathbf{F M}_{\mathcal{M} \times \mathcal{E}}(\mathbf{Z})$ and $(\ldots, 1,1,1, \ldots)$ is the $1 \times \mathcal{M}$ matrix in which all entries are equal to 1 , then $(\ldots, 1,1,1, \ldots) A$ is a $1 \times \mathcal{E}$-matrix with at most finitely many non-zero entries, that is, $(\ldots, 1,1,1, \ldots) A \in \mathbf{Z}^{(\mathcal{E})}$. Similarly, if $A^{t}$ is the transpose of $A$ and $(\ldots, 1,1,1, \ldots)$ is the $1 \times \mathcal{E}$-matrix in which all entries are equal to 1 , then $(\ldots, 1,1,1, \ldots) A^{t}$ is a $1 \times \mathcal{M}$-matrix with at most finitely many non-zero entries, that is, $(\ldots, 1,1,1, \ldots) A^{t} \in \mathbf{Z}^{(\mathcal{M})}$. Hence there is a homomorphism of abelian groups $\chi: \mathbf{F M}_{\mathcal{M} \times \mathcal{E}}(\mathbf{Z}) \rightarrow \mathbf{Z}^{(\mathcal{M})} \oplus \mathbf{Z}^{(\mathcal{E})}$ defined by $\chi: A \mapsto\left((\ldots, 1,1,1, \ldots) A^{t},(\ldots, 1,1,1, \ldots) A\right)$.

Finally, from Theorem 1.9 it follows that there is a homomorphism of abelian groups $\psi: \operatorname{Groth}\left(\mathcal{S}_{R}\right) \rightarrow \mathbf{Z}^{(\mathcal{M})} \oplus \mathbf{Z}^{(\mathcal{E})}$ defined by $\psi([U])=\left([U]_{m},[U]_{e}\right)$ for every non-zero uniserial $R$-module $U$.

Theorem 3.1. (a) The diagram

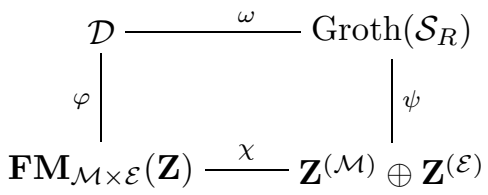

is commutative.

(b) The mapping $\psi$ is injective.

(c) The monoids $\operatorname{Groth}\left(\mathcal{S}_{R}\right)^{+}$and $\chi(F)$ are isomorphic.

(d) The Grothendieck group $\operatorname{Groth}\left(\mathcal{S}_{R}\right)$ of serial $R$-modules of finite Goldie dimension is a free abelian group.

Proof. The commutativity of the diagram is obvious. Let $[M]-[N]$ be an arbitrary element of $\operatorname{Groth}\left(\mathcal{S}_{R}\right), M, N \in \mathcal{S}_{R}$, and suppose $[M]-[N] \in \operatorname{ker} \psi$. Then $M=U_{1} \oplus \cdots \oplus U_{r}$ and $N=V_{1} \oplus \cdots \oplus V_{s}$ for suitable non-zero uniserial modules $U_{1}, \ldots, U_{r}, V_{1}, \ldots, V_{s}$, and $\psi\left(\left[U_{1}\right]\right)+\cdots+\psi\left(\left[U_{r}\right]\right)=\psi\left(\left[V_{1}\right]\right)+\cdots+\psi\left(\left[V_{s}\right]\right)$, so that $r=s,\left[U_{1}\right]_{m}, \ldots,\left[U_{r}\right]_{m}$ is a permutation of $\left[V_{1}\right]_{m}, \ldots,\left[V_{s}\right]_{m}$, and $\left[U_{1}\right]_{e}, \ldots,\left[U_{r}\right]_{e}$ is a permutation of $\left[V_{1}\right]_{e}, \ldots,\left[V_{s}\right]_{e}$. From Theorem 1.9 we get that $U_{1} \oplus \cdots \oplus U_{r} \cong$ $V_{1} \oplus \cdots \oplus V_{s}$, and thus $[M]=[N]$ and $\psi$ is injective. From the commutativity of the diagram in (a), we get that $\operatorname{Groth}\left(\mathcal{S}_{R}\right)^{+} \cong \psi\left(\operatorname{Groth}\left(\mathcal{S}_{R}\right)^{+}\right)=\psi \omega(\mathcal{D})=\chi \varphi(\mathcal{D})=$ $\chi(F)$. Finally, $\psi$ injective and $\mathbf{Z}^{(\mathcal{M})} \oplus \mathbf{Z}^{(\mathcal{E})}$ free imply $\operatorname{Groth}\left(\mathcal{S}_{R}\right)$ free.

The submonoid $\operatorname{Groth}\left(\mathcal{S}_{R}\right)^{+}$is the positive cone for a natural partial order $\leq$in $\operatorname{Groth}\left(\mathcal{S}_{R}\right)$. Explicitly, one has $[A]-[B] \leq[C]-[D]$ if and only if there exists $E \in \mathcal{S}_{R}$ such that $A \oplus D \oplus E \cong B \oplus C$. The abelian group $\mathbf{Z}^{(\mathcal{M})} \oplus \mathbf{Z}^{(\mathcal{E})}$ also has a natural partial order, the pointwise partial order, in which, for $(f, g),\left(f^{\prime}, g^{\prime}\right) \in \mathbf{Z}^{(\mathcal{M})} \oplus \mathbf{Z}^{(\mathcal{E})}$, 
we have $(f, g) \leq\left(f^{\prime}, g^{\prime}\right)$ if and only if $f\left([V]_{m}\right) \leq f^{\prime}\left([V]_{m}\right)$ and $g\left([V]_{e}\right) \leq g^{\prime}\left([V]_{e}\right)$ for every non-zero uniserial module $V$. The mapping $\psi: \operatorname{Groth}\left(\mathcal{S}_{R}\right) \rightarrow \mathbf{Z}^{(\mathcal{M})} \oplus \mathbf{Z}^{(\mathcal{E})}$ is only an injective morphism of partially ordered abelian groups, so that we can deduce that $\operatorname{Groth}\left(\mathcal{S}_{R}\right)$ is a free abelian group, but we cannot deduce that it is necessarily order isomorphic to a free abelian group with the pointwise order. This explains why Krull-Schmidt can fail for serial modules.

\section{REFERENCES}

[1] R. Camps and W. Dicks, On semilocal rings, Israel J. Math. 81 (1993), 203-211. MR 94m:16027

[2] A. Facchini, D. Herbera, L. S. Levy and P. Vámos, Krull-Schmidt fails for artinian modules, Proc. Amer. Math. Soc. 123 (1995), 3587-3592. MR 96b:16020

[3] A. Facchini and L. Salce, Uniserial modules: sums and isomorphisms of subquotients, Comm. Algebra 18(2) (1990), 499-517. MR 91h:16005

[4] D. Herbera and A. Shamsuddin, Modules with semi-local endomorphism ring, Proc. Amer. Math. Soc. 123 (1995), 3593-3600. MR 96b:16014

[5] I. Kaplansky, Elementary divisors and modules, Trans. Amer. Math. Soc. 66 (1949), 464491. MR 11:155b

[6] B. Stenström, Rings of Quotients, Springer-Verlag, Berlin, 1975.

[7] K. Varadarajan, Dual Goldie dimension, Comm. Algebra 7 (1979), 565-610. MR 80d:16014

[8] R. B. Warfield, Serial rings and finitely presented modules, J. Algebra 37 (1975), 187-222. MR 53:5663

Dipartimento di Matematica e Informatica, Università di Udine, 33100 Udine, Italy

E-mail address: facchini@dimi.uniud.it 